\title{
The Psychosocial Impact of Testing Individuals with No History of Genital Herpes for Herpes Simplex Virus Type 2
}

TOMIO MIYAI, MPH, KATHERINE R. TURNER, MPH, CHARLOTTE K. KENT, MPH, AND

JEFFREY KLAUSNER, MD, MPH

\begin{abstract}
Background: Although approximately $20 \%$ of the population has a genital herpes (HSV-2) infection, $80 \%$ of these infections are unrecognized or asymptomatic. Serologic identification of HSV-2 leads to recognition of infection, which could lead to behavioral changes that reduce transmission. However, there has been concern that HSV-2 testing among persons without symptoms will cause substantial psychosocial harm.

Goal: The goal of this study was to assess the psychosocial impact of an HSV-2 diagnosis among individuals without a history of genital herpes attending a sexually transmitted disease (STD) clinic.

Study: We conducted a cohort study of persons with no history of genital herpes attending an STD clinic and seeking herpes testing. Two follow-up interviews were conducted 1 week and 3 months after persons received their test results. Serum was tested using HerpeSelect 2. Psychosocial morbidity was assessed at baseline and each follow up using a mental health score, sexual attitude score, and perception of genital herpes score.
\end{abstract}

Results: Twenty-one percent (41 of 196) of participants tested positive for HSV-2 antibody. Among patients who were HSV-2-positive, there was no significant change in mental health score from baseline during either follow-up visit, nor was there any difference compared with persons who were HSV-2-negative. Patients who were HSV-2-positive did have a decline $(P=0.01)$ in their sexual attitude scores at the 1-week follow up compared with persons who were HSV-2-negative, indicating a decrease in positive sexual attitude, but this difference no longer remained at the 3-month follow up $(P=0.74)$. Patients who were HSV-2-positive viewed having genital herpes as significantly less traumatic than patients who were HSV-2-negative at both follow-up visits $(P<0.01)$.

Conclusion: There was no apparent lasting adverse psychosocial impact of detecting HSV-2 infection among individuals without a history of genital herpes seeking herpes testing at an STD clinic.

ALTHOUGH GENITAL HERPES IS one of the most common sexually transmitted diseases (STDs) in the United States (22\% prevalence), ${ }^{1,2}$ studies indicate that $80 \%$ of herpes infections are unrecognized or asymptomatic. ${ }^{1}$ The development and commercial availability of reliable type-specific serologic assays that distinguish between herpes simplex virus type 1 (HSV-1) and type 2 (HSV-2) provide the opportunity to identify previously undiagnosed HSV-2 infection, the common cause of genital herpes.

This research was supported by the San Francisco Department of Public Health.

Correspondence: Charlotte K. Kent, MPH, STD Prevention and Control Services, 1360 Mission St., Suite 401, San Francisco, CA 94103. E-mail: Charlotte.kent@sfdph.org

Received for publication September 24, 2003, revised April 21, 2004, and accepted April 26, 2004.
From STD Prevention and Control Services, San Francisco Department of Public Health, San Francisco, California

Strategies to reduce genital herpes transmission include avoiding contact when herpes lesions are present, condom use, and suppressive therapy. ${ }^{3,4}$ However, these strategies require an individual to know about their herpes infection. Serologic HSV-2 testing leads to diagnosis of infection. Once individuals are aware of their infection, counseling and education can lead to approximately half of these individuals recognizing their herpes symptoms, and they can be further counseled to avoid contact when lesions are present. ${ }^{5,6}$ However, because half of serologically diagnosed individuals do not report symptoms, and because transmission often occurs during periods of asymptomatic viral shedding, counseling these individuals about behavior change such as condom use can reduce transmission to a seronegative partner regardless of whether symptoms are present or a person recognizes their symptoms. ${ }^{3}$

Although serologic diagnosis of HSV-2 infection along with counseling and education could reduce HSV-2 transmission, the value of serologic testing is debated..$^{7-10}$ A primary argument against serologic HSV-2 testing is that testing individuals without a history of genital herpes will cause psychosocial harm. ${ }^{8}$ Few studies to date have assessed the psychosocial impact of a serologic HSV-2 diagnosis. The only previously published study conducted to evaluate this issue found no immediate or lasting psychosocial burden among patients who tested HSV-2-positive. ${ }^{11}$

The municipal STD clinic in San Francisco began offering type-specific serologic testing for HSV-2 in 2000 to patients who specifically requested genital herpes testing. The routine STD testing offered at the clinic includes chlamydia, gonorrhea, and syphilis, but not HSV-2. Before adding serologic HSV-2 testing to the routine STD tests, clinicians felt it important to assess the psychosocial impact of a serologic HSV-2 diagnosis.

The objective of this study was to assess the psychosocial impact of a positive HSV-2 diagnosis among individuals without a history of genital herpes attending the STD clinic. Psychosocial impact was evaluated by measuring changes in mental health and well-being, sexual attitudes, and perception of genital herpes following serologic HSV-2 testing.

\section{Methods}

\section{Study Population}

The study was conducted at the San Francisco municipal STD clinic between April and August 2001. Patients specifically asking 
clinicians for genital herpes testing were informed of this study and asked to participate. Eligible patients were fluent Englishspeakers, over 18 years of age, had no history of genital herpes symptoms, had no prior clinical diagnosis of genital herpes, were willing to have their blood drawn, and were able to give written informed consent. Patients with symptomatic infections, or who were diagnosed with genital herpes in the past, were not eligible for the study. The local Institutional Review Board approved the protocol.

\section{Study Design}

Study staff administered a baseline interview after informed consent was given. A study nurse obtained a blood sample from the participant for HSV-2 type-specific serologic testing. Patients received results by calling the clinic's results line. Patients testing positive for HSV-2 were provided standardized counseling by experienced study staff. Two follow-up telephone interviews were conducted 1 week (follow-up 1) and 3 months (follow-up 2) after a participant received his or her test results.

\section{Laboratory Methods}

Serum samples were tested for HSV-2 antibodies by the HerpeSelect 2 (Focus Technologies, Cypress, CA) enzyme-linked immunosorbent assay (ELISA) at the public health laboratory. The test uses recombinant HSV-2 gG2 antigen to identify the HSV-2 antibody and has a reported sensitivity of $97.7 \% .{ }^{12}$

\section{Psychosocial Morbidity}

We designed a questionnaire to assess psychosocial morbidity at 3 time points: during the baseline interview, at follow-up 1 (1week follow up), and follow-up 2 (3-month follow up). The questionnaire collected information on demographics, sexual behavior, mental health, sexual attitudes, and perception of genital herpes. We modified 2 questionnaires to assess mental health and sexual attitudes. We assessed mental health using a modified version of the Rand Mental Health Inventory 5 item version (MHI-5). The MHI-5 evaluates emotional well-being, anxiety, depression, and behavioral/emotional control. ${ }^{13}$ We modified the 6 -point scale $(1=$ all of the time or always; $6=$ none of the time or never) to a 5 -point scale $(1=$ never; $5=$ always $)$ that allowed participants to pick a neutral response $(3=$ sometimes $)$. The
Multidimensional Sexual Self-Concept Questionnaire (MSSCQ) is a comprehensive100-item survey that explores 20 aspects of sexual self-concept. ${ }^{14}$ To assess sexual attitudes for this study, we took 10 questions from the survey that only assessed sexual anxiety, sexual optimism, sexual self-monitoring, sexual satisfaction, sexual fear/apprehension, and sexual depression. We also revised the 5 -point scale responses from "not at all characteristic of me" (1) and "very characteristic of me" (5) to "strongly disagree" (1) and "strongly agree." (5) Finally, we evaluated perception of genital herpes by administering an instrument from the American Social Health Association (ASHA) that assessed perceived trauma associated with genital herpes; responses were on a 4-point scale ranging from "not traumatic at all" (1) to "very traumatic" (4). ${ }^{15}$

\section{Data Analysis}

We entered data into a computerized database and analyzed the data using EpiInfo v6.04 (version 6.04; Centers for Disease Control and Prevention, Atlanta, GA). We calculated prevalence rate ratios and $95 \%$ confidence intervals (CIs) to examine any statistical associations between HSV-2 serostatus and baseline demographic characteristics. At each time point, we calculated a mean score for mental health, sexual attitudes, and perception of genital herpes. We used Student $t$ tests to determine any significant changes from zero (no change) over time in the mental health score, sexual attitudes score, and perception of genital herpes from baseline to 1-week follow up and 3-month follow up. We used Student $t$ tests to evaluate if changes in mental health, sexual attitudes, and perception of genital herpes from baseline to follow up differed significantly by HSV-2 serostatus. We also dichotomized changes in mental health scores between the baseline visit and each follow-up visit by persons who had no change or an improvement in mental health score compared with persons who had a decrease in mental health scores. We then calculated risk ratios and $95 \%$ CIs.

\section{Results}

\section{Characteristics of Study Patients}

One hundred ninety-six patients of the 201 individuals completing the baseline questionnaire were eligible to participate in the study. The 5 ineligible patients were excluded because they had

Table 1. Demographic Characteristics of Study Population by HSV-2 Status

\begin{tabular}{|c|c|c|c|c|}
\hline Variable & $\mathrm{N}$ & HSV-2 + No. (\%) & $\mathrm{RR}$ & $95 \% \mathrm{Cl}$ \\
\hline \multicolumn{5}{|l|}{ Gender/sexual orientation } \\
\hline Heterosexual men & 86 & $15(17.4)$ & Reference & \\
\hline Women & 34 & 7 (20.6) & 1.2 & $0.5-2.6$ \\
\hline \multicolumn{5}{|l|}{ Age } \\
\hline $18-20$ & 84 & $12(14.3)$ & Reference & \\
\hline $30-39$ & 75 & $16(21.3)$ & 1.5 & $0.8-3.0$ \\
\hline White/caucasian & 117 & $19(16.2)$ & Reference & \\
\hline African-American & 28 & $12(42.9)$ & 2.6 & $1.5-4.8$ \\
\hline Other & 50 & $9(18.0)$ & 1.1 & $0.5-2.3$ \\
\hline \multicolumn{5}{|l|}{ Education } \\
\hline$>$ Some college & 151 & $26(17.2)$ & Reference & \\
\hline$<$ High school & 45 & $15(33.3)$ & 1.9 & $1.1-3.3$ \\
\hline
\end{tabular}


equivocal results (2), never completed the blood draw (1), already knew their HSV-2 status (1), or were HSV-2-negative but had genital herpes resulting from HSV-1 (1). One hundred eighty (92\%) patients completed the first follow-up interview, and 158 (81\%) completed the second follow-up interview. Patients who tested positive for HSV-2 were less likely to complete the second follow-up interview $(P=0.07)$. Eighty-seven percent (36 of 41) of HSV-2-positive participants completed the first follow-up interview, and $70 \%$ (29 of 41) completed their second interview, compared with $93 \%$ (144 of 155) at first follow up and 83\% (129 of 155) at second follow up of HSV-2-negative participants. Patients did not differ by follow-up status in terms of gender, age, sexual orientation, or income. Patients with less than or equal to a high school education were less likely to complete the first follow-up interview $(P=0.04)$ and the second follow-up interview $(P<0.01)$.

\section{Herpes Simplex Virus Type 2 Serostatus}

Forty-one (20.9\%) study patients tested positive for HSV-2 antibody. Testing positive for HSV-2 antibody was not associated with gender, sexual orientation, or income. Presence of HSV-2 antibody was associated with increasing age $(P=0.03)$, being black $(P<0.01)$, and lower education level $(P=0.02)$ (Table 1$)$.

\section{Psychosocial Morbidity}

Mental Health. Among patients testing positive for HSV-2, there was no significant decline or improvement in the mental health score from baseline to 1-week follow up $(P=0.44)$, or from baseline to 3 -month follow up $(P=0.60)$ (Table 2). HSV-2negative patients demonstrated improvement on their mental health score at 1 -week follow up $(P<0.01)$ and at 3 -month follow up $(P=0.03)$ (Table 2). However, when compared with each other, there were no differences in mental health scores between patients who were HSV-2-positive and those who were HSV-2 negative from baseline to 1 -week follow up $(P=0.48)$ or 3-month follow up $(P=0.58)$, although HSV-2-positive patients' scores were $8 \%$ to $9 \%$ lower at the 2 follow-up visits than persons who were HSV-2-negative (Table 2). When we dichotomized persons by having a decrease in mental health scores compared with no change or an improvement in mental health scores, persons who were HSV-2-positive were as likely to have a decrease in mental health scores as persons who were HSV-2-negative at both the first follow-up visit (relative risk [RR], 1.1; 95\% CI, 0.7-1.9) and the second follow-up visit (RR, 1.0; 95\% CI, 0.6-1.7).

Sexual Attitudes. There were no changes in the sexual attitudes score among HSV-2-positive or HSV-2-negative individuals from baseline to 1 -week follow up (HSV-2-positive: $P=0.11$; HSV2-negative: $P=0.18$ ) or 3-month follow up (HSV-2-positive: $P=$ 0.89 ; HSV-2-negative: $P=0.14$ ). However, when compared with each other, HSV-2-positive individuals scored lower than HSV-2negative patients at the 1 -week follow up $(P=0.01)$, indicating a decrease in positive sexual attitudes. This difference no longer existed at the 3 -month follow up $(P=0.74)$.

Perception of Genital Herpes. At baseline, there was no difference in the perception of genital herpes between patients who were HSV-2-seropositive and -seronegative, with 35\% (14 of 40) of patients who were HSV-2-positive and 35.5\% (55 of 155) of patients who were HSV-2-negative perceiving genital herpes as "very traumatic." After receiving their HSV-2 antibody results, only $14 \%$ (5 of 36 ) and $10 \%$ (3 of 29 ) of individuals who were HSV-2-positive reported genital herpes as "very traumatic" at

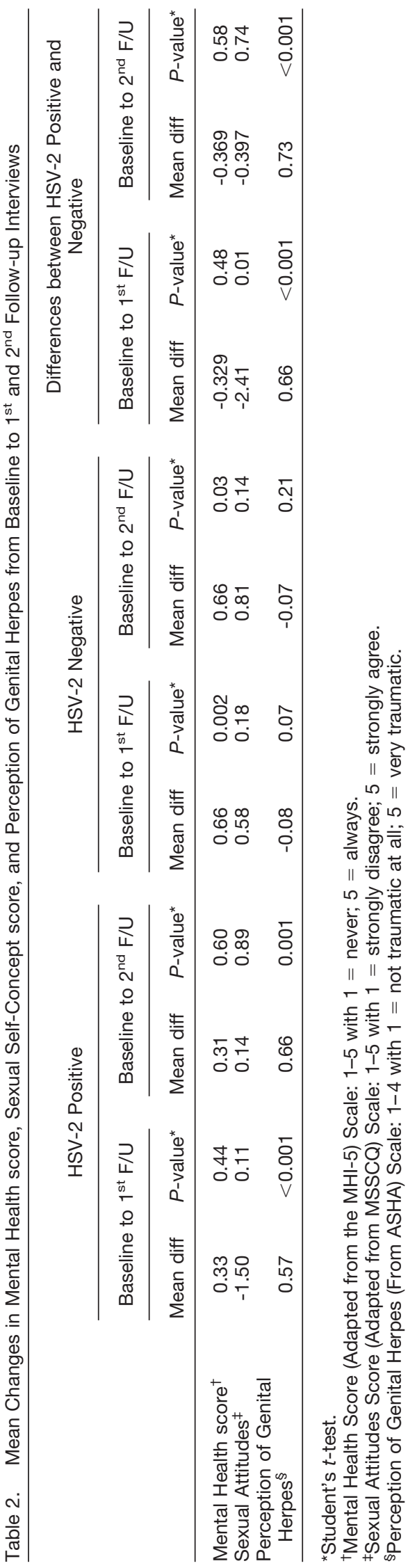


1-week follow up and 3-month follow up, respectively. The perception that genital herpes was less traumatic postdiagnosis among individuals who were HSV-2-positive was significant at both follow-up time points $(P<0.01)$ (Fig. 1). The proportion of patients who were HSV-2-negative and who viewed genital herpes as "very traumatic" increased slightly at 1 -week follow up $(P=0.07)$, but there was no change at the 3 -month follow up $(P=0.21)$. Patients who were HSV-2-positive viewed having genital herpes as less traumatic than patients who were HSV-2-negative at both follow-up time points $(P<0.001)$.

\section{Discussion}

Our results indicate that there was no lasting adverse psychosocial morbidity among individuals without a history of genital herpes who received a positive serologic HSV-2 diagnosis. Although individuals who were HSV-2-negative had improved mental health scores after their negative diagnosis, the individuals who were HSV-2-positive had no change in their mental health status, suggesting no adverse impact to mental health among these individuals. There was a decline in sexual attitude scores among participants who were HSV-2-positive at the first followup. Although this normalizes at the second follow up, this suggests a temporary impact on the participants that may be critically important to the individuals, and could influence their sexual and social relationships. Lastly, patients diagnosed with HSV-2 infection viewed having genital herpes as less traumatic than they did before their diagnosis.

A number of factors could contribute to the lack of psychosocial morbidity associated with a positive HSV-2 diagnosis found in this study. First, individuals who were HSV-2-positive were somewhat more likely to be lost to follow up. Individuals who were HSV2-positive who were lost to follow up could have been more severely affected as a result of their diagnosis. The lack of any further associations between HSV-2 infection and adverse psychosocial indicators could also be influenced by the lower follow-up rate among individuals who were HSV-2-positive. Second, patients received thorough pretest counseling regarding genital herpes, and among infected patients, there was intensive posttest counseling and education. These counseling and education sessions could ameliorate a patient's adverse response to an HSV-2 diagnosis. Third, study patients had no history of genital herpes symptoms. For these individuals, their experience with the infection could be very different from a person with a symptomatic infection who has painful lesions that are visible to partners and recur often. Our study suggests that persons infected with HSV-2, but who do not have classic herpes symptoms, might not experience lasting adverse psychosocial morbidity associated with their infection.

There are several limitations to this study. First, we used a convenience sample of STD clinic patients who had no history of genital herpes and who specifically requested herpes testing. The results of this study cannot be generalized to other populations of persons without a history of genital herpes, or to other populations that are not seeking STD services. Secondly, the small sample size and lower HSV-2 prevalence than expected, reduced the power of the study and could contribute to a lack of significant associations. We only had sufficient power to detect a difference in mental health scores of $50 \%$ or more between HSV-2-positive and HSV2-negative persons. Third, the instruments we used to assess psychosocial morbidity might not have been sensitive enough to capture true psychosocial burden. The study was not designed to measure a person's mental health status, or report whether a person was in good or poor mental health; rather, we wanted to measure changes over time and examine differences between groups. We felt that these instruments were adequate at detecting differences had there been a true association. Finally, as explained in the "Methods" section, the MHI-5 and the MSSCQ instruments were modified from their original and validated versions. Although these modifications did not impact our ability to measure changes over time or between groups, we cannot compare our results with other studies that have used these validated instruments.

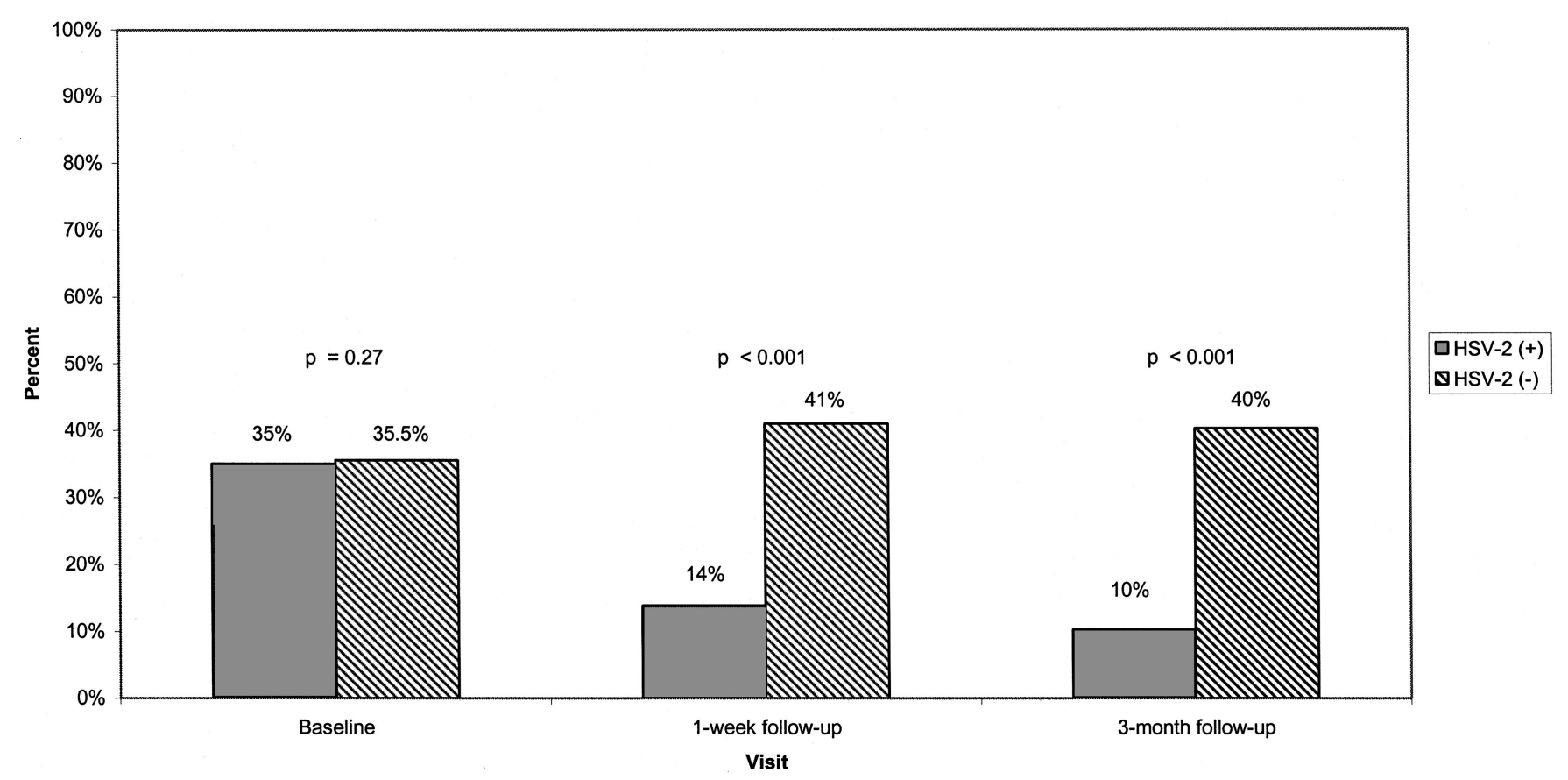

Fig. 1. Percent of patients who view having genital herpes infection as "very traumatic" at baseline and first and second follow-up visits. 
The diagnosis of HSV-2 among individuals with unrecognized infections has public health implications because it could lead to the reduction of HSV-2 transmission. STD clinics are an ideal setting to introduce serologic HSV-2 testing and to provide related counseling and education. Our study indicates that there was no lasting adverse psychosocial morbidity associated with a positive serologic HSV-2 diagnosis. Concern about causing psychosocial morbidity among asymptomatic individuals seeking HSV testing should not be a barrier to offering testing at STD clinics. Given the fixed availability of resources at STD clinics, routine HSV-2 screening might not be feasible. However, we recommend that serologic HSV-2 tests be available to STD clinic attendees and suggest that with proper counseling and education, there will be minimal psychosocial impact on asymptomatic persons with a positive serologic test.

\section{References}

1. Fleming DT, McQuillin GM, Johnson RE, et al. Herpes simplex virus type 2 in the United States, 1975 to 1994. N Engl J Med 1997; $337: 1105-1111$.

2. Corey L, Handsfield H. Genital herpes and public health: Addressing a global problem. JAMA 2000; 283:791-794.

3. Wald A, Langenberg A, Link K, et al. Effect of condoms on reducing the transmission of herpes simplex virus type 2 from men to women. JAMA 2001; 285:3100-3106.

4. Corey L, Wald A, Patel R, et al. Once-daily valacyclovir to reduce the transmission of genital herpes. N Engl J Med. 2004;350:11-20.

5. Wald A, Zeh J, Selke $\mathrm{S}$, et al. Reactivation of genital herpes simplex virus type 2 infection in asymptomatic seropositive persons. $\mathrm{N}$ Engl J Med 2000; 342:844-850.

6. Langenberg A, Benedetti J, Jenkins J, et al. Development of clinically recognizable genital lesions among women previously identified as having 'asymptomatic' herpes simplex type 2 infection. Ann Intern Med 1989; 110:882-887.

7. Patrick DM, Money D. Debate: The argument for. Should every STD clinic patient be considered for type-specific serological screening for HSV? Herpes 2002; 9:32-34.

8. Mindel A, Taylor J. Debate: the argument against: Should every STD clinic patient be considered for type-specific serological screening for HSV? Herpes 2002; 9:35-37.

9. Brown ZA. HSV-2 specific serology should be offered routinely to antenatal patients. Rev Med Virol 2000; 10:141-144.

10. Wilkinson D, Barton S, Cowan F. HSV-2 specific serology should not be offered routinely to antenatal patients. Rev Med Virol 2000; $10: 145-153$.

11. Smith A, Denham I, Keogh L, et al. Psychosocial impact of typespecific herpes simplex serological testing on asymptomatic sexual health clinic attendees. Int J STD AIDS 2000; 11:15-20.

12. Turner KR, Wong EH, Kent CK, et al. Serologic herpes testing in the real world: validation of new type-specific serologic herpes simplex virus tests in a public health laboratory. Sex Transm Dis 2002; 29:422-425.

13. Berwick DM, Murphy JM, Goldman PA, et al. Performance of a five-item mental health screening test. Med Care 1991; 29:169-176.

14. Snell WE, Fisher TD, Walters AS. The Multidimensional Sexuality Questionnaire: An objective self-report measure of psychological tendencies associated with human sexuality. In Snell WE Jr, ed. New Directions in the Psychology of Human Sexuality: Research and Theory. Cape Giradeau, MO: Snell Publications. Available at: http:// cstl-cla.semo.edu/snell/books/sexuality/sexuality.htm. 2001.

15. Schulz SL, Ebel CW, Alexander L. What Americans think about genital herpes: Differences by race. Poster Presentation, National STD Prevention Conference; Milwaukee, WI; December 7-10, 2000 . 